\title{
Highly-dispersive mirrors reach new levels of dispersion
}

\author{
Elena Fedulova, ${ }^{1, *}$ Kilian Fritsch, ${ }^{2}$ Jonathan Brons, ${ }^{1}$ Oleg Pronin, ${ }^{1,2}$ \\ Tatiana Amotchkina, ${ }^{3}$ Michael Trubetskov, ${ }^{1,3}$ Ferenc Krausz, ${ }^{1,2}$ and Vladimir Pervak ${ }^{2,4}$ \\ ${ }^{1}$ Max Planck Institute of Quantum optics, Hans-Kopfermann str. 1, 85748, Garching, Germany \\ ${ }^{2}$ Ludwig Maximilians University, Am Coulombwall 1, 85748, Garching, Germany \\ ${ }^{3}$ Research Computing Center, Moscow State University, Leninskie Gory, 119992, Moscow, Russia \\ ${ }^{4}$ Ultrafast Innovations GmbH, Am Coulombwall 1, 85748 Garching, Germany \\ EElena.Fedulova@mpq.mpg.de
}

\begin{abstract}
A highly-dispersive mirror with the unprecedented group delay dispersion of $-10000 \mathrm{fs}^{2}$ in the wavelength range of $1025-1035 \mathrm{~nm}$ is reported. Reproducible production of a coating with such a high dispersion was possible due to the recently developed robust synthesis technique. Successful employment of the new highly-dispersive mirror in an oscillator is demonstrated.
\end{abstract}

(C)2015 Optical Society of America

OCIS codes: (310.4165) Multilayer design; (310.1620) Interference coatings; (320.5520) Pulse compression.

\section{References and links}

1. R. Szipöcs, K. Ferencz, C. Spielmann, and F. Krausz, "Chirped multilayer coatings for broadband dispersion control in femtosecond lasers," Opt. Lett. 19(3), 201-203 (1994).

2. V. Pervak, C. Teisset, A. Sugita, S. Naumov, F. Krausz, and A. Apolonski, "High-dispersive mirrors for femtosecond lasers," Opt. Express 16(14), 10220-10233 (2008).

3. P. Dombi, P. Rácz, M. Lenner, V. Pervak, and F. Krausz, "Dispersion management in femtosecond laser oscillators with highly dispersive mirrors," Opt. Express 17(22), 20598-20604 (2009).

4. V. Pervak, O. Pronin, O. Razskazovskaya, J. Brons, I. B. Angelov, M. K. Trubetskov, A. V. Tikhonravov, and F. Krausz, "High-dispersive mirrors for high power applications," Opt. Express 20(4), 4503-4508 (2012).

5. C. R. E. Baer, C. Kränkel, C. J. Saraceno, O. H. Heckl, M. Golling, R. Peters, K. Petermann, T. Südmeyer, G. Huber, and U. Keller, "Femtosecond thin-disk laser with 141 W of average power," Opt. Lett. 35(13), 2302$2304(2010)$.

6. D. Bauer, I. Zawischa, D. H. Sutter, A. Killi, and T. Dekorsy, "Mode-locked Yb:YAG thin-disk oscillator with $41 \mu \mathrm{J}$ pulse energy at $145 \mathrm{~W}$ average infrared power and high power frequency conversion," Opt. Express 20(9), 9698-9704 (2012).

7. J. Brons, V. Pervak, E. Fedulova, D. Bauer, D. Sutter, V. Kalashnikov, A. Apolonskiy, O. Pronin, and F. Krausz, "Energy scaling of Kerr-lens mode-locked thin-disk oscillators," Opt. Lett. 39(22), 6442-6445 (2014).

8. V. Pervak, V. Fedorov, Y. A. Pervak, and M. Trubetskov, "Empirical study of the group delay dispersion achievable with multilayer mirrors," Opt. Express 21(15), 18311-18316 (2013).

9. A. V. Tikhonravov and M. K. Trubetskov, "OptiLayer software," http://www.optilayer.com.

10. A. V. Tikhonravov, M. K. Trubetskov, and G. W. Debell, "Application of the needle optimization technique to the design of optical coatings," Appl. Opt. 35(28), 5493-5508 (1996).

11. A. V. Tikhonravov, M. K. Trubetskov, and G. W. DeBell, "Optical coating design approaches based on the needle optimization technique," Appl. Opt. 46(5), 704-710 (2007).

12. A. V. Tikhonravov and M. K. Trubetskov, "Modern design tools and a new paradigm in optical coating design," Appl. Opt. 51(30), 7319-7332 (2012).

13. V. Pervak, M. K. Trubetskov, and A. V. Tikhonravov, "Robust synthesis of dispersive mirrors," Opt. Express 19(3), 2371-2380 (2011).

14. D. Ristau, H. Ehlers, T. Gross, and M. Lappschies, "Optical broadband monitoring of conventional and ion processes," Appl. Opt. 45(7), 1495-1501 (2006).

15. W. H. Knox, N. M. Pearson, K. D. Li, and C. A. Hirlimann, "Interferometric measurements of femtosecond group delay in optical components," Opt. Lett. 13(7), 574-576 (1988).

16. W. H. Knox, "Dispersion measurements for femtosecond-pulse generation and applications," Appl. Phys. B 58(3), 225-235 (1994).

17. A. Gosteva, M. Haiml, R. Paschotta, and U. Keller, "Noise-related resolution limit of dispersion measurements with white-light interferometers,” J. Opt. Soc. Am. B 22(9), 1868-1874 (2005).

\#235910 - \$15.00 USD Received 10 Mar 2015; revised 5 May 2015; accepted 6 May 2015; published 18 May 2015 
18. T. V. Amotchkina, A. V. Tikhonravov, M. K. Trubetskov, D. Grupe, A. Apolonski, and V. Pervak, "Measurement of group delay of dispersive mirrors with white-light interferometer," Appl. Opt. 48(5), 949-956 (2009).

19. J. C. Diels and W. Rudolph, Ultrafast Laser Pulse Phenomena (Academic Press, 2006), Chap. 9.

\section{Introduction}

Highly-dispersive mirrors (HDMs) have become a key tool in the field of ultrafast physics over the last two decades [1-4]. Presently, the majority of femtosecond lasers include dispersive mirror optics which allow precise control of group delay dispersion (GDD) characteristics.

Due to its power scalability, thin-disk (TD) technology may constitute the basis for nextgeneration femtosecond laser oscillators, yielding average output powers exceeding $140 \mathrm{~W}$ $[5,6]$ and pulse energies of $40 \mu \mathrm{J}[6]$, with pulse durations of 700-1000 fs.

More recently, a power scalable laser setup, where an Yb:YAG TD oscillator delivered an average output power of $270 \mathrm{~W}$, corresponding to $14.4 \mu \mathrm{J}$ pulse energy at $18.8 \mathrm{MHz}$ repetition rate in the mode-locked regime, has been presented [7]. Mode-locked operation in the regime of anomalous dispersion was realized by 8 bounces each on two HD folding mirrors with a GDD of $-3000 \mathrm{fs}^{2}$ each [4], introducing a total roundtrip GDD of $-48000 \mathrm{fs}^{2}$.

Scaling passively-mode-locked femtosecond laser oscillators to ever higher pulse energies calls for ever higher negative GDD in the cavity. Meeting this requirement with an increasing number of bounces off dispersive mirrors tends to increase alignment sensitivity, round-trip losses, and complexity of the system. Advancing femtosecond oscillator technology to higher peak as well as average powers relies on the development of HDMs with higher levels of dispersion.

Generally, the larger the required bandwidth, the smaller the achievable mean value of GDD in dispersive mirrors [2,4]. Recent studies [8] have shown that there are certain limitations for the maximum achievable negative value of GDD, which depend on the total thickness of coatings and the layer material combination. For multilayer structures based on the material pair $\mathrm{Ta}_{2} \mathrm{O}_{5} / \mathrm{SiO}_{2}$ this value reaches $-7000 \mathrm{fs}^{2}$ for a thickness of $9.8 \mu \mathrm{m}$. Today, state-of-the-art coating technology allows to produce coatings with total physical thicknesses larger than $10 \mu \mathrm{m}$ and to reach even higher levels of GDD. Here we report achievement of the maximum possible negative GDD allowed by this total design thickness and its successful application in an $\mathrm{Yb}: Y A G$ TD oscillator.

\section{Design considerations}

A commercial OptiLayer software package [9] was used to design an HDM with high negative dispersion. The software utilizes most powerful approaches such as needle optimization and gradual evolution algorithms [10-12], enabling one to avoid problems of convergence at a local minimum.

An implementation of a single HDM with a GDD of $-10000 \mathrm{fs}^{2}$ is a challenge due to the high sensitivity of the design performance to manufacturing errors. In view of this fact additional considerations had to be made. A method to circumvent manufacturing errors is presented in [13], which can be considered as a generalization of the highly efficient needle optimization and gradual evolution techniques [10-12]. The described robust synthesis procedure was used in this work.

\#235910 - \$15.00 USD Received 10 Mar 2015; revised 5 May 2015; accepted 6 May 2015; published 18 May 2015 (C) 2015 OSA 1 Jun 2015 | Vol. 23, No. 11 | DOI:10.1364/OE.23.013788 | OPTICS EXPRESS 13789 


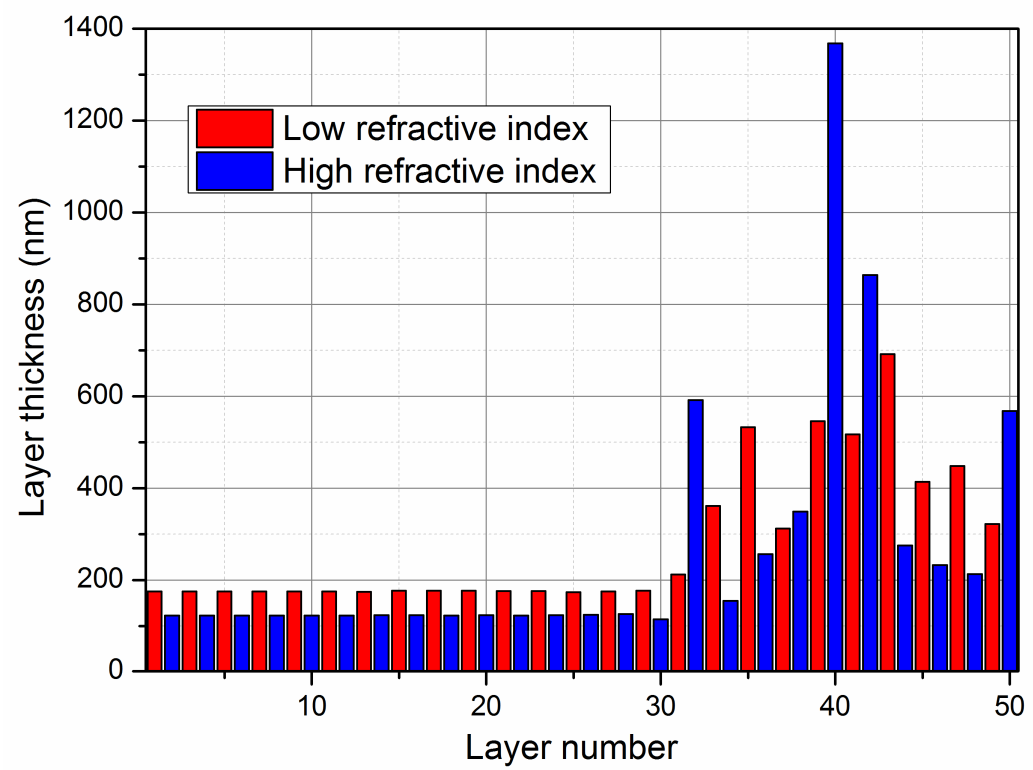

Fig. 1. Physical thicknesses of the individual layers of the HDM. Red bars represent the low refractive index material $\mathrm{SiO}_{2}$. Blue bars represent the high index material $\mathrm{Ta}_{2} \mathrm{O}_{5}$. The layers are displayed starting from a substrate. The bottom layer \#1 is closest to the substrate, the top layer \#50 is closest to the incident medium (air).

The target design was optimized to have a GDD of $-10000 \mathrm{fs}^{2}$ and reflectance of $100 \%$ for the wavelength range 1025-1035 nm. According to [8], where the limits for the maximum achievable negative value of the GDD have been studied, the target GDD of $-10000 \mathrm{fs}^{2}$ is achievable for the specified spectral range and for the chosen thin film materials with state-ofthe-art deposition technology. The layer thicknesses of the optimized multilayer structure are shown in Fig. 1. Dependencies of the refractive indices on the wavelength are described by the Cauchy formula:

$$
n(\lambda)=n_{\infty}+A / \lambda^{2}+B / \lambda^{4}
$$

where $\lambda$ is the wavelength expressed in microns, $n_{\infty}, A$ and $B$ are coefficients presented in Table 1.

Table 1. Cauchy formula coefficients for the substrate and layer materials.

\begin{tabular}{llll}
\hline & $n_{\infty}$ & $A$ & $B$ \\
\hline Suprasil & 1.4433 & $4.06 \mathrm{E}-3$ & $6.94818 \mathrm{E}-6$ \\
$\mathrm{SiO}_{2}$ & 1.4653 & 0.0 & $4.71080 \mathrm{E}-4$ \\
$\mathrm{Ta}_{2} \mathrm{O}_{5}$ & 2.0657 & $1.6830 \mathrm{E}-2$ & $1.686 \mathrm{E}-3$ \\
\hline
\end{tabular}

The obtained via the described above robust procedure design consists of 15 pairs of alternating quarter-wave layers (corresponding to the central wavelength of $1030 \mathrm{~nm}$ ) of $\mathrm{Ta}_{2} \mathrm{O}_{5}$ and $\mathrm{SiO}_{2}$, the material pair which is usually used for low loss coatings with relatively small bandwidths (200-300 nm), and 20 chirped top layers, resulting in 50 layers and a total thickness of $13.7 \mu \mathrm{m}$.

\section{Production process}

The designed multilayer structure was produced by use of the magnetron sputtering technique (Helios, Leybold Optics) equipped with broadband in situ monitoring (BBM) [14]. The plant 
is equipped with two proprietary TwinMags magnetrons and a plasma source for plasma/ionassisted reactive middle-frequency dual-magnetron sputtering. It exploits precise timecontrolled monitoring of layer thicknesses, based on careful calibration of deposition process parameters affecting deposition rates, with additional control provided by the BBM system. A Suprasil substrate was used as a test witness sample during the process. Layers at deposition rates of $0.4 \mathrm{~nm} / \mathrm{s}$ allow for rapid manufacturing as well as the production of dense layers (near intrinsic solid density) with excellent homogeneity and high reproducibility.

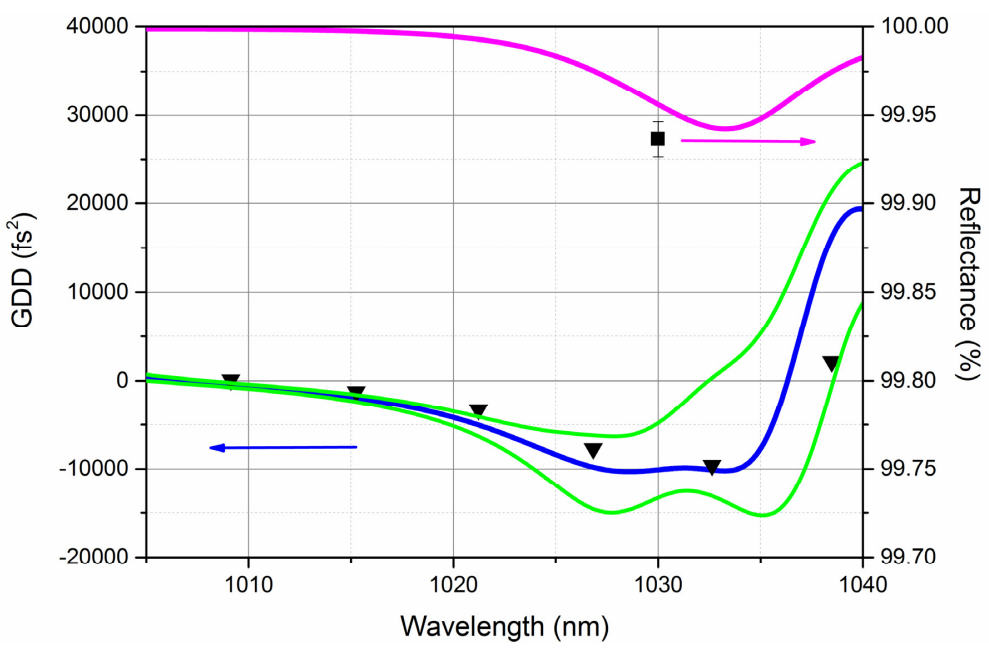

Fig. 2. Comparison of the designed and measured data for the new HDM: The designed GDD for $3^{\circ}$ angle of incidence (blue curve), error bars $\pm 0.5 \mathrm{~nm}$ (green curves); the measurement performed with a WLI at $3^{\circ}$ angle of incidence (black triangles). The designed reflectance for $7.5^{\circ}$ angle of incidence (magenta curve), the square at $1030 \mathrm{~nm}$ represents the measurement performed with a lossmeter at $7.5^{\circ}$ angle of incidence.

The produced samples were characterized with the help of a home-built white-light interferometer (WLI) [15-18] and a lossmeter based on a cavity ring-down technique (NovaWave Technologies LossPro). The comparison of the designed and measured data is presented in Fig. 2, revealing a good agreement between theory and experiment. Most importantly, the agreement is particularly good in the relevant $(1025-1035 \mathrm{~nm})$ wavelength range. The result proves the feasibility of robust HDMs with negative GDD relying on physical thicknesses in excess of $10 \mu \mathrm{m}$.

The main benefit of the HDM developed in the present study is the possibility to obtain high negative GDD in a single optical element. The HDM reported here operates perfectly in a laser system and exhibits total losses of $600 \mathrm{ppm}$. HDMs having a GDD of $-4000 \mathrm{fs}^{2}$ with total losses of $1000 \mathrm{ppm}$ and a GDD of $-3000 \mathrm{fs}^{2}$ with total losses of $300 \mathrm{ppm}$ were demonstrated in [4]. The HDM reported here possesses over twice the GDD without the expense of introducing further losses.

\section{HDM in Yb:YAG oscillator}

Here we report the first application of the newly-created HDM, as a source of GDD required to implement stable Kerr-lens mode-locking (KLM) in an Yb:YAG TD laser. In order to test the new HDM 4 standard DMs from [2] (3 bounces on $-3000 \mathrm{fs}^{2}$ mirrors and 1 bounce on $1000 \mathrm{fs}^{2}$ mirror in reference setup) were replaced by 3 highly-reflective (HR) mirrors and the new HDM (test setup), providing the same amount of GDD, namely $-20000 \mathrm{fs}^{2}$ per roundtrip.

The oscillator used was a basic diode-pumped Yb:YAG TD oscillator in which modelocking was achieved by hard-aperture KLM with a 2-mm sapphire crystal serving as the nonlinear Kerr medium (Fig. 3). The oscillator was operated in the mode-locked regime at a repetition rate of $33.7 \mathrm{MHz}$ with an average output power of approximately $4 \mathrm{~W}$.

\#235910 - \$15.00 USD Received 10 Mar 2015; revised 5 May 2015; accepted 6 May 2015; published 18 May 2015 


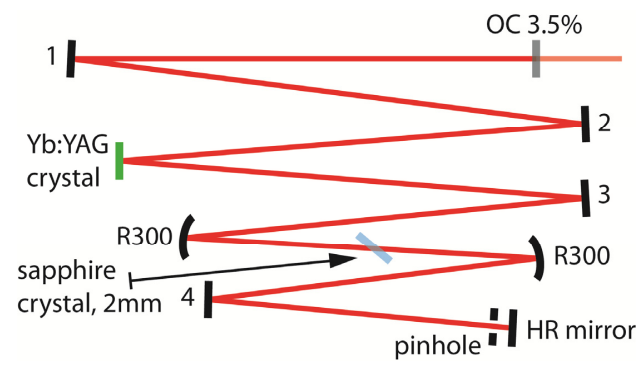

Fig. 3. Schematic of the oscillator built for testing the intra cavity behavior of the new HDM. The oscillator is a basic diode-pumped KLM Yb:YAG TD laser. OC, output coupler. R300, HR mirrors with radius of curvature $300 \mathrm{~mm}$. Mode-locking was achieved by hard aperture KLM with the sapphire crystal and a pinhole. Mirrors 1 to 4 were either a set of 4 known DMs with a total roundtrip GDD of $-20000 \mathrm{fs}^{2}$ or 3 HR mirrors and the new HDM with the same amount of GDD. During the measurements the oscillator ran at $33.7 \mathrm{MHz}$ repetition rate with an output power of $\sim 4 \mathrm{~W}$.

The spectrum and the autocorrelation of the pulses were measured for both setups. Figure 4 provides evidence that the measured curves fit the expected $\operatorname{sech}^{2}-$ function well. The spectra are centered around a wavelength of $1030.5 \mathrm{~nm}$ with a full-width at half-maximum (FWHM) of $4.2 \mathrm{~nm}$ in the reference case and $3.9 \mathrm{~nm}$ in the test case. Pulse durations of $290 \mathrm{fs}$ and $320 \mathrm{fs}$ in the reference and test setup respectively were calculated from their autocorrelation via $\tau_{p}=\tau_{a c} / 1.543$ [19], where $\tau_{p}$ is the FWHM of the pulse intensity and $\tau_{a c}$ is the FWHM of the intensity autocorrelation. Since realignment of the cavity between the two experiments is unavoidable it cannot be concluded that the slight difference in spectral width and pulse duration is solely attributed to the new HDM.

The advantage of having only one mirror providing the GDD is that fewer mirrors can be used to build a working cavity. This improves the stability and simplifies the alignment since fewer degrees of freedom need to be taken into account and fewer surfaces are prone to optical damage. The use of fewer mirrors also establishes the possibility to build shorter cavities with higher repetition rates.
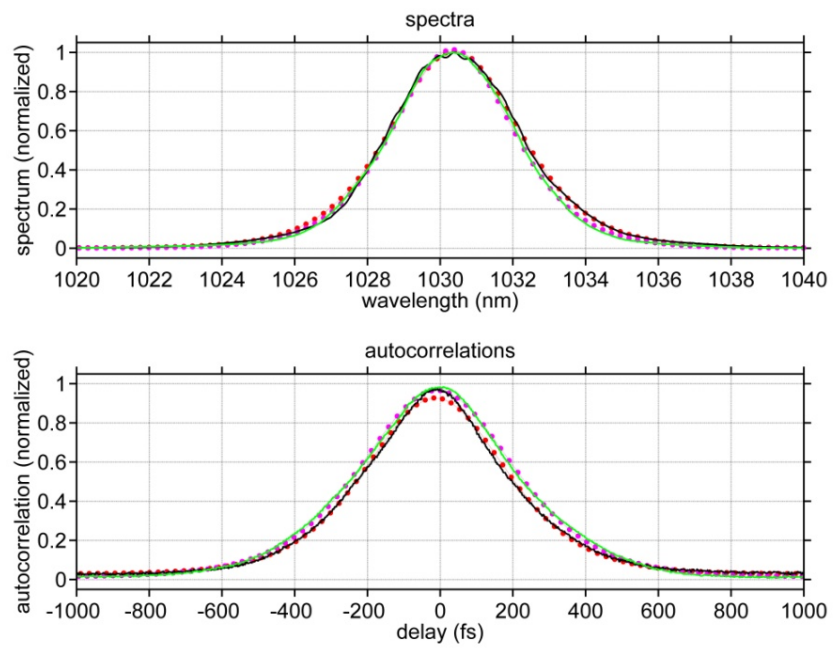

Fig. 4. Comparison of spectra (top) and autocorrelations (bottom) of a basic KLM Yb:YAG oscillator working with the known mirror set and with the new HDM. In both graphs the color coding as follows: measured data with reference setup (black line), measured data with test setup (green line), fitted $\operatorname{sech}^{2}$-function to reference data (red dots), fitted $\mathrm{sech}^{2}$-function to test data (magenta dots).

\#235910 - \$15.00 USD Received 10 Mar 2015; revised 5 May 2015; accepted 6 May 2015; published 18 May 2015 
It is important to mention that the rather large thickness of the produced coating may lead to a reduction in the damage threshold or to thermal effects, such as expansion and deformation of the coating at higher intra cavity powers. HDMs with a GDD of $-10000 \mathrm{fs}^{2}$ possessing higher damage thresholds and negligible thermal effects are in the process of development.

\section{Conclusion}

A single HDM with a GDD reaching the value of $-10000 \mathrm{fs}^{2}$ in the wavelength range 1025$1035 \mathrm{~nm}$ has been presented for the first time. The recently reported robust technique has been used in order to circumvent manufacturing errors and to produce a challenging, rather thick $(13.7 \mu \mathrm{m})$ coating with such a large negative GDD for central wavelength of $1030 \mathrm{~nm}$. The produced HDM was successfully utilized in an Yb:YAG TD oscillator, operating at 33.7 $\mathrm{MHz}$ repetition rate with an output power of about $4 \mathrm{~W}$, which resulted in a pulse duration of $320 \mathrm{fs}$.

Advancing HDMs to ever higher negative dispersion benefits the development of compact, user-friendly, high-power femtosecond oscillators and may even open the prospect for simplifying pulse stretching-compression schemes in chirped-pulse amplifier systems.

\section{Acknowledgments}

This work was supported by the Cluster of Excellence "Munich-Centre for Advanced Photonics" (http://www.munich-photonics.de/) of the German Research Foundation (DFG).

\#235910 - \$15.00 USD Received 10 Mar 2015; revised 5 May 2015; accepted 6 May 2015; published 18 May 2015 